\title{
Guidelines for institutions granting bariatric privileges for the use of laparoscopic techniques
}

\author{
Bruce Schirmer
}

Published online: 17 October 2010

(C) Springer Science+Business Media, LLC 2010

The authors of this document and the Society of American Gastrointestinal Endoscopic Surgeons (SAGES) Guidelines Committee as a whole deserve praise for the prudent, comprehensive, and fair nature of their guidelines. The aim of the guidelines is clearly stated at the beginning of the document, and it must be emphasized that this document should not be misinterpreted in legal venues as representing the standard of care. Rather, it is a suggested guideline for hospital committees attempting to determine the appropriate granting of privileges to surgeons wishing to perform laparoscopic bariatric surgery.

Guidelines must always balance the extremes of being too arduous or too lenient. Excessively arduous guidelines limit access to care, prevent potentially competent practitioners from performing a needed service, and may, in selected politically oriented situations, attempt to create monopolies of practice based on discipline, background, or other specific criteria. Conversely, excessively lenient guidelines potentially compromise patient safety and outcomes by allowing inexperienced practitioners to perform procedures without sufficient training or support to ensure likely optimal outcomes.

This document proposes guidelines that are well detailed in terms of previous training and experience, with careful emphasis on a defined difference between bariatric operations that involve organ division with anastomosis creation (e.g., Roux-en-Y gastric bypass) and those that do not (e.g., laparoscopic adjustable gastric banding). The skill sets for performing the two operations are slightly different, with the former requiring more advanced overall laparoscopic

B. Schirmer $(\bowtie)$

Department of Surgery, University of Virginia Health System, Charlottesville, VA, USA

e-mail: BS@hscmail.mcc.virginia.edu skills as well as experience in organ division and reanastomosis.

The document also recognizes the fact that performing laparoscopic bariatric surgery successfully involves two major areas of experience and skill: bariatric surgery itself and advanced laparoscopic surgery. The latter may be a skill developed without any bariatric experience, and the former requires such experience. The competent laparoscopic bariatric surgeon must have both skill sets. Individuals who require training in both certainly need more time and training than those well versed in one skill set but not the other.

Probably, the safer of those two scenarios is that of the competent bariatric surgeon who must learn advanced laparoscopic surgery. Reliance on conversion to familiar open surgery during the learning curve of that surgeon's advanced laparoscopic skill acquisition likely is safer than the reverse situation in which a skilled laparoscopic surgeon is performing an entirely new operation with a new set of patient selection, perioperative risk factor, and patient care parameters.

This author has been impressed over the years by the usual highly careful and considerate approach of surgeons performing new procedures. During the introduction of laparoscopic surgery, although a few individual surgeons were not so careful and caused an increase in the adverse outcomes for such procedures (e.g., bile duct injuries after laparoscopic cholecystectomy), those individuals were by far in the minority. Community surgeons whose local reputation is highly fragile, based on potentially adverse outcomes, usually were extremely cautious and proceeded only in a team fashion with adequate preparation for new procedures. Academic institutions also usually provided mentoring and assistance by more experienced faculty to prevent early complications. 
Despite all these efforts, we do know that the early outcomes in any individual's experience likely will be less excellent than those after considerable experience. Guidelines are a suggested formula for preventing significant adverse outcomes and promoting a rapid learning curve. Outcomes, however, need to be vigilantly monitored by credentialing committees in all circumstances when a novice practitioner is performing a new procedure. We currently suffer from the fact that the requirements for privileging and those for other more lofty goals, such as being designated as a "qualified bariatric surgeon" by accrediting bodies for centers of excellence, are based on numbers of procedures alone, without adequate outcomes data parameters. In the future, the acquisition of current data from national data banks at these centers of excellence networks and from national research studies will potentially supply outcome parameters for determining whether practitioners are proceeding safely with the introductory phase of their experiences. These numbers, however, will not substitute for the published guidelines that serve as a basis upon which the initial privileges to begin such an experience are given.

As a final note, surgeons who begin their laparoscopic bariatric surgery experience should, even if they more than meet the recommended criteria in these guidelines, exercise judicious caution in patient selection and choice of operation. High-risk medical patients, excessively large patients, and patients with previous upper abdominal operations all should be avoided early in one's experience with laparoscopic bariatric surgery. Certainly, elective revisional bariatric surgery for failed weight loss has no place in the early experience of a laparoscopic bariatric surgeon. The lack of correlating physical examination, the diminished organ reserve, and the potential for rapid deterioration of this patient population when adversity occurs all make this field a distinct challenge for the surgeon.

Nevertheless, the ongoing epidemic of obesity and its severe comorbid medical problems are not going away. Despite the current best efforts of the insurance companies (in the United States) to make the operation beyond the reach of many with typical health insurance, more than 23 million people in the United States alone and many more internationally would benefit from laparoscopic bariatric surgery. This patient population currently is woefully underserved, and the medical discrimination against them is likely to end in the future on the basis of societal needs alone. Therefore, these guidelines, as an effort to safely increase the number of bariatric surgeons to meet the inevitable future need, are laudatory and appropriate.

Disclosures Bruce Schirmer has no conflicts of interest or financial ties to disclose. 